\title{
A note to the convergence rates in precise asymptotics
}

Jianjun $\mathrm{He}^{*}$

"Correspondence: hejj@cjlu.edu.cn Department of Mathematics, China Jiliang University, Hangzhou, 310018, China

\begin{abstract}
Let $\left\{X, X_{n}, n \geq 1\right\}$ be a sequence of i.i.d. random variables with zero mean. Set $S_{n}=\sum_{k=1}^{n} X_{k}, E X^{2}=\sigma^{2}>0$, and $\lambda_{r, p}(\epsilon)=\sum_{n=1}^{\infty} n^{r / p-2} P\left(\left|S_{n}\right| \geq n^{1 / p} \epsilon\right)$. In this paper, the author discusses the rate of approximation of $\frac{p}{r-p} E|N|^{2(r-p) /(2-p)}$ by $\epsilon^{2(r-p) /(2-p)} \lambda_{r, p}(\epsilon)$ under suitable moment conditions, where $N$ is normal with zero mean and variance $\sigma^{2}>0$, which improves the results of Gut and Steinebach (J. Math. Anal. Appl. 390:1-14, 2012) and extends the work He and Xie (Acta Math. Appl. Sin. 29:179-186, 2013). Specially, for the case $r=2$ and $p=\frac{1}{\beta+1}, \beta>-\frac{1}{2}$, the author discusses the rate of approximation of $\frac{\sigma^{2}}{2 \beta+1}$ by $\epsilon^{2} \lambda_{2,1 /(\beta+1)}(\epsilon)$ under the condition $E X^{2} /(|X|>t)=O\left(t^{-\delta} /(t)\right)$ for some $\delta>0$, where $/(t)$ is a slowly varying function at infinity.

MSC: $60 \mathrm{~F} 15 ; 60 \mathrm{G} 50$
\end{abstract}

Keywords: convergence rate; precise asymptotics; slowly varying function

\section{Introduction}

Let $\left\{X, X_{n}, n \geq 1\right\}$ be a sequence of i.i.d. random variables. Set $S_{n}=\sum_{k=1}^{n} X_{k}$ and $\lambda_{r, p}(\epsilon)=$ $\sum_{n=1}^{\infty} n^{r / p-2} P\left(\left|S_{n}\right| \geq n^{1 / p} \epsilon\right)$. Heyde [1] proved that

$$
\lim _{\epsilon \rightarrow 0} \epsilon^{2} \lambda_{2,1}(\epsilon)=\sigma^{2}
$$

whenever $E X=0$ and $E X^{2}=\sigma^{2}<\infty$. Klesov [2] studied the rate of the approximation of $\sigma^{2}$ by $\epsilon^{2} \lambda_{2,1}(\epsilon)$ under the condition $E|X|^{3}<\infty$. He and Xie [3] improved the results of Klesov [2]. Gut and Steinebach [4] extended the results of Klesov [2] and obtained the following Theorem $A$. Gut and Steinebach [5] studied the general idea of proving precise asymptotics.

Theorem A Let $\left\{X, X_{n}, n \geq 1\right\}$ be a sequence of i.i.d.random variables with zero mean and $0<p<2, r \geq 2$.

(1) If $E X^{2}=\sigma^{2}>0$ and $E|X|^{q}<\infty$ for some $r<q \leq 3$, then

$$
\epsilon^{2(r-p) /(2-p)} \lambda_{r, p}(\epsilon)-\frac{p}{r-p} E|N|^{2(r-p) /(2-p)}=o\left(\epsilon^{\frac{p(q-2)(r-p)}{(q-p)(2-p)}}\right) .
$$

(2) If $E X^{2}=\sigma^{2}>0$ and $E|X|^{q}<\infty$ for some $q \geq 3$ with $q>\frac{2 r-3 p}{2-p}$, then

$$
\epsilon^{2(r-p) /(2-p)} \lambda_{r, p}(\epsilon)-\frac{p}{r-p} E|N|^{2(r-p) /(2-p)}=o\left(\epsilon^{\frac{2 p(r-p)}{(2-p)(p+2 q-p q)}}\right),
$$

where $N$ is normal with mean 0 and variance $\sigma^{2}>0$.

๑ $2013 \mathrm{He}$; licensee Springer. This is an Open Access article distributed under the terms of the Creative Commons Attribution License (http://creativecommons.org/licenses/by/2.0), which permits unrestricted use, distribution, and reproduction in any medium, provided the original work is properly cited. 
The purpose of this paper is to strengthen Theorem $A$ and extend the theorem of He and Xie [3] under suitable moment conditions. In addition, we shall discuss the rate at which $\epsilon^{2} \lambda_{2,1 /(\beta+1)}(\epsilon)$ converges to $\frac{\sigma^{2}}{2 \beta+1}$ under the condition $T(t)=O\left(t^{-\delta} l(t)\right)$ for some $\delta>0$, where $T(t)=E X^{2} I(|X|>t), l(t)$ is a slowly varying function at infinity. Throughout this paper, $C$ represents a positive constant, though its value may change from one appearance to the next, and $[x]$ denotes the integer part of $x . \Phi(x)$ is the standard normal distribution function, $\Phi(x)=\frac{1}{\sqrt{2 \pi}} \int_{-\infty}^{x} e^{-t^{2} / 2} d t, \varphi(x)=\Phi^{\prime}(x)$.

\section{Main results}

From Gut and Steinebach [6], it is easy to obtain the following lemma.

Lemma 2.1 Let $\left\{X, X_{n}, n \geq 1\right\}$ be a sequence of i.i.d. normal distribution random variables with zero mean and variance $\sigma^{2}>0$. Set $0<p<2$ and $r \geq 2$, then

$$
\begin{gathered}
\epsilon^{2(r-p) /(2-p)} \sum_{n=1}^{\infty} n^{(r / p)-2} P\left(\left|S_{n}\right| \geq n^{1 / p} \epsilon\right)-\frac{p}{r-p} E|N|^{2(r-p) /(2-p)} \\
\quad= \begin{cases}O\left(\epsilon^{2(r-p) /(2-p)}\right), & r<3 p, \\
O\left(\epsilon^{4 p /(2-p)}\right), & r \geq 3 p .\end{cases}
\end{gathered}
$$

Lemma 2.2 (Bingham et al. [7]) Let $l(t)$ be a slowly varying function. We have

(1) for any $\eta>0$,

$$
\lim _{t \rightarrow \infty} t^{\eta} l(t)=\infty, \quad \lim _{t \rightarrow \infty} t^{-\eta} l(t)=0
$$

(2) if $0<\delta<1$, then

$$
\int_{a}^{t} s^{-\delta} l(s) d s \sim \frac{1}{1-\delta} t^{1-\delta} l(t), \quad t \rightarrow \infty
$$

(3) if $\delta>1$, then

$$
\int_{t}^{\infty} s^{-\delta} l(s) d s \sim-\frac{1}{1-\delta} t^{1-\delta} l(t), \quad t \rightarrow \infty
$$

(4) if $\delta=1$, then $L(t)=\int_{t}^{\infty} \frac{l(s)}{s} d s, m(t)=\int_{a}^{t} \frac{l(s)}{s} d s$ are slowly varying functions; and

$$
\lim _{t \rightarrow \infty} \frac{l(t)}{L(t)}=0, \quad \lim _{t \rightarrow \infty} \frac{l(t)}{m(t)}=0 .
$$

Theorem 2.1 Let $\left\{X, X_{n}, n \geq 1\right\}$ be a sequence of i.i.d.random variables with zero mean and $0<p<2, r \geq 2$.

(1) If $E X^{2}=\sigma^{2}>0$ and $E|X|^{3}<\infty$ for some $r<3$, then

$$
\epsilon^{2(r-p) /(2-p)} \lambda_{r, p}(\epsilon)-\frac{p}{r-p} E|N|^{2(r-p) /(2-p)}= \begin{cases}O\left(\epsilon^{2(r-p) /(2-p)}\right), & 2 \leq r<\frac{3 p}{2} \\ O\left(\epsilon^{p /(2-p)} \log \frac{1}{\epsilon}\right), & r=\frac{3 p}{2} \\ O\left(\epsilon^{p /(2-p)}\right), & \frac{3 p}{2}<r<3\end{cases}
$$


(2) If $E X^{2}=\sigma^{2}>0$ and $E|X|^{2+\delta}<\infty$ for some $0<\delta<1, r<2+\delta$, then

$$
\begin{aligned}
& \epsilon^{2(r-p) /(2-p)} \lambda_{r, p}(\epsilon)-\frac{p}{r-p} E|N|^{2(r-p) /(2-p)} \\
& \quad= \begin{cases}O\left(\epsilon^{2(r-p) /(2-p)}\right), & 2 \leq r<(1+\delta / 2) p, \\
O\left(\epsilon^{p \delta /(2-p)} \log \frac{1}{\epsilon}\right), & r=(1+\delta / 2) p, \\
o\left(\epsilon^{p \delta /(2-p)}\right), & (1+\delta / 2) p<r<2+\delta .\end{cases}
\end{aligned}
$$

(3) If $E X^{2}=\sigma^{2}>0$ and $E|X|^{q}<\infty$ for some $q \geq 3$ with $q>\frac{2 r-3 p}{2-p}$, then

$$
\epsilon^{2(r-p) /(2-p)} \lambda_{r, p}(\epsilon)-\frac{p}{r-p} E|N|^{2(r-p) /(2-p)}= \begin{cases}O\left(\epsilon^{2(r-p) /(2-p)}\right), & 2 \leq r<\frac{3 p}{2} \\ O\left(\epsilon^{p /(2-p)} \log \frac{1}{\epsilon}\right), & r=3 p / 2, \\ O\left(\epsilon^{p /(2-p)}\right), & r>3 p / 2\end{cases}
$$

where $N$ is normal with mean 0 and variance $\sigma^{2}>0$.

Remark 2.1 Clearly, Theorem 1 and Theorem 2 in He and Xie [3] are special cases of Theorem 2.1, by taking $r=2$ and $p=1$.

Remark 2.2 If $0<p<2, r \geq 2$, we have $\min \left(\frac{2(r-p)}{2-p}, \frac{p \delta}{2-p}\right)>\frac{p \delta(r-p)}{(2+\delta-p)(2-p)}$ for $r<2+\delta=q \leq 3$ and $\min \left(\frac{2(r-p)}{2-p}, \frac{p}{2-p}\right)>\frac{2(r-p) p}{(2-p)(p+2 q-p q)}$ for some $q \geq 3$ with $q>\frac{2 r-3 p}{2-p}$. So, the results of Theorem 2.1 are stronger than those of Theorem A.

Theorem 2.2 Let $\left\{X, X_{n} ; n \geq 1\right\}$ be a sequence of i.i.d random variables with zero mean, and let $T(t)=O\left(t^{-\delta} l(t)\right)$ for some $\delta>0$, where $l(t)$ is a slowly varying function at infinity. Set $E X^{2}=\sigma^{2}>0$ and $\beta>-\frac{1}{2}$.

(1) If $\delta>1$, then

$$
\epsilon^{2} \lambda_{2,1 /(\beta+1)}(\epsilon)-\frac{\sigma^{2}}{2 \beta+1}= \begin{cases}O\left(\epsilon^{2}\right), & -\frac{1}{2}<\beta<-\frac{1}{4} \\ O\left(\epsilon^{2} \log \frac{1}{\epsilon}\right), & \beta=-\frac{1}{4} \\ O\left(\epsilon^{1 /(2 \beta+1)}\right), & \beta>-\frac{1}{4}\end{cases}
$$

(2) If $0<\delta<1$, then

$$
\epsilon^{2} \lambda_{2,1 /(\beta+1)}(\epsilon)-\frac{\sigma^{2}}{2 \beta+1}= \begin{cases}O\left(\epsilon^{2}\right), & -\frac{1}{2}<\beta<-\frac{1}{2}+\frac{\delta}{4} \\ O\left(\epsilon^{\delta /(2 \beta+1)} l\left(\epsilon^{-1 /(2 \beta+1)}\right)\right), & \beta \geq-\frac{1}{2}+\frac{\delta}{4}\end{cases}
$$

(3) If $\delta=1$, then

$$
\epsilon^{2} \lambda_{2,1 /(\beta+1)}(\epsilon)-\frac{\sigma^{2}}{2 \beta+1}= \begin{cases}O\left(\epsilon^{2}+\epsilon^{2} \int_{1}^{\epsilon^{-1 /(2 \beta+1)}} \frac{l(t)}{t} d t\right), & -\frac{1}{2}<\beta<-\frac{1}{4}, \\ O\left(\epsilon^{2}\left(1+\int_{1}^{\epsilon^{-5}} \frac{l(t)}{t} d t\right) \log \frac{1}{\epsilon}\right), & \beta=-\frac{1}{4}, \\ O\left(\epsilon^{1 /(2 \beta+1)}\left(1+\int_{1}^{\epsilon^{-(2 \beta+3) /(2 \beta+1)}} \frac{l(t)}{t} d t\right)\right), & \beta>-\frac{1}{4} .\end{cases}
$$

Remark 2.3 For $r=2, p=\frac{1}{\beta+1}$. If $l(t)=1$, then the result of Theorem 2.2 is weaker than that of Theorem 2.1 for $0<\delta<1, \beta \geq-\frac{1}{2}+\frac{\delta}{4}$, and weaker than that of Theorem 2.1 for 
$\delta=1$. But the condition $T(t)=O\left(t^{-\delta}\right)$ is weaker than the condition $E|X|^{2+\delta}<\infty$. If $l(t) \rightarrow 0$ as $t \rightarrow \infty$, then the result of Theorem 2.2 is the same as that of Theorem 2.1 for $0<\delta<1$.

Remark 2.4 For $\delta>0$, the condition $E|X|^{2+\delta}<\infty$ is neither sufficient nor necessary for the condition $T(t)=O\left(t^{-\delta} l(t)\right)$. Here are some suitable examples.

Example 1 Let $X$ be a random variable with density $f(x)=\frac{C(1+\delta \ln |x|)}{|x|^{3+\delta} \ln ^{2}|x|} I(|x|>e)$, where $C$ is a normalizing constant, and $0<\delta<1$, then $E X=0$ and $T(t)=\frac{C}{t^{\delta} \ln t} I(t>e), l(t)=\frac{1}{\ln t}$ is a slowly varying function at infinity. But $E|X|^{2+\delta}=C \int_{|x|>e} \frac{1+\delta \ln |x|}{|x| \ln ^{2}|x|} d x=\infty$.

Example 2 Let $X$ be a random variable with density $f(x)=\frac{C\left(\delta \ln ^{2}|x|+|x|(\ln |x|-1)\right)}{|x|^{\delta+3} \ln ^{2}|x| e^{|x|} / \ln |x|} I(|x|>e)$, where $0<\delta<1$, then $E X=0$ and $T(t)=\frac{C}{t^{\delta} e^{t / \ln (t)}} I(t>e), h(t)=\frac{1}{e^{t / \ln t}}, E|X|^{2+\delta}<\infty$. But $h(t)=\frac{1}{e^{t / \ln t}}$ is not a slowly varying function at infinity.

In fact, we have the following result.

Theorem 2.3 Suppose $X$ is a real random variable and $\delta>0$. Then $E|X|^{2+\delta}<\infty$ if and only if $t^{\delta} T(t) \rightarrow 0$ and $\int_{t}^{\infty} s^{\delta-1} T(s) d s \rightarrow 0$ as $t \rightarrow \infty$.

Remark 2.5 If $t^{\delta} T(t)$ is bounded as $t \rightarrow \infty$ for some $\delta>0$, then we have $E|X|^{2+\alpha}<\infty$ for every $\alpha \in(0, \delta)$ from Theorem 2.3 .

Remark 2.6 Let $X$ be a random variable with zero mean. If there exist positive constants $C_{1}$ and $C_{2}$ such that $C_{1} l(t) \leq t^{\delta} T(t) \leq C_{2} l(t)$ for sufficiently large $t$ and some $\delta>0$, where $l(t)$ is a slowly varying function at infinity, then from Lemma 2.2(4) and Theorem 2.3, we have

$$
E|X|^{2+\delta}<\infty \quad \Leftrightarrow \quad \int_{t}^{\infty} \frac{l(s)}{s} d s \rightarrow 0 \quad \text { as } t \rightarrow \infty .
$$

\section{Proofs of the main results}

Proof of Theorem 2.1 Without loss of generality, we suppose that $\sigma^{2}=1,0<\epsilon<1$. Since

$$
P\left(\left|S_{n}\right| \geq n^{1 / p} \epsilon\right)=2\left(1-\Phi\left(n^{(2-p) / 2 p} \epsilon\right)\right)+R_{n}
$$

where

$$
R_{n}=P\left(S_{n} \leq-n^{1 / p} \epsilon\right)-\Phi\left(-n^{1 / p-1 / 2} \epsilon\right)+\Phi\left(n^{1 / p-1 / 2} \epsilon\right)-P\left(S_{n} \leq n^{1 / p} \epsilon\right) .
$$

From (3.1), we have

$$
\begin{aligned}
& \epsilon^{2(r-p) /(2-p)} \lambda_{r, p}(\epsilon)-\frac{p}{r-p} E|N|^{2(r-p) /(2-p)} \\
& =2 \epsilon^{2(r-p) /(2-p)} \sum_{n=1}^{\infty} n^{r / p-2}\left(1-\Phi\left(n^{(2-p) / 2 p} \epsilon\right)\right)-\frac{p}{r-p} E|N|^{2(r-p) /(2-p)} \\
& \quad+\epsilon^{2(r-p) /(2-p)} \sum_{n=1}^{\infty} n^{r / p-2} R_{n} .
\end{aligned}
$$


By Lemma 2.1, in order to prove Theorem 2.1, we only need to estimate $\epsilon^{2(r-p) /(2-p)} \times$ $\sum_{n=1}^{\infty} n^{r / p-2} R_{n}$.

(1) On account of a non-uniform estimate of the central limit theorem by Nagaev [8], for every $x \in R$,

$$
\left|P\left(\frac{S_{n}}{\sqrt{n}}<x\right)-\Phi(x)\right| \leq \frac{C E|X|^{3}}{\sqrt{n}(1+|x|)^{3}} .
$$

By (3.3), $\left|R_{n}\right| \leq \frac{C E|X|^{3}}{\sqrt{n}\left(1+\epsilon n^{(2-p) / 2 p)^{3}}\right.}$.

(a) If $r<3 p / 2$, then

$$
\epsilon^{2(r-p) /(2-p)} \sum_{n=1}^{\infty} n^{r / p-2} R_{n} \leq C \epsilon^{2(r-p) /(2-p)} \sum_{n=1}^{\infty} n^{r / p-5 / 2}=O\left(\epsilon^{2(r-p) /(2-p)}\right) .
$$

(b) If $3 p / 2<r<3$, then

$$
\begin{aligned}
& \epsilon^{2(r-p) /(2-p)} \sum_{n=1}^{\infty} n^{r / p-2} R_{n} \\
& \quad \leq C \epsilon^{2(r-p) /(2-p)} \sum_{n=1}^{\infty} \frac{n^{r / p-2}}{\sqrt{n}\left(1+\epsilon n^{(2-p) / 2 p}\right)^{3}} \\
& \quad \leq C \epsilon^{2(r-p) /(2-p)}\left(\sum_{n=1}^{\left[\epsilon^{-2 p /(2-p)}\right]} \frac{n^{r / p-2}}{\sqrt{n}}+\epsilon^{-3} \sum_{n=\left[\epsilon^{-2 p /(2-p)}\right]+1}^{\infty} n^{r / p-5 / 2-(6-3 p) / 2 p}\right) \\
& =O\left(\epsilon^{p /(2-p)}\right) .
\end{aligned}
$$

(c) If $r=3 p / 2$, then

$$
\begin{aligned}
\epsilon^{2(r-p) /(2-p)} \sum_{n=1}^{\infty} n^{r / p-2} R_{n} & \leq C \epsilon^{p /(2-p)}\left(\sum_{n=1}^{\left[\epsilon^{-2 p /(2-p)}\right]} \frac{1}{n}+\epsilon^{-3} \sum_{n=\left[\epsilon^{-2 p /(2-p)]+1}\right.}^{\infty} n^{-1-(6-3 p) / 2 p}\right) \\
& =O\left(\epsilon^{p /(2-p)} \log \frac{1}{\epsilon}\right) .
\end{aligned}
$$

From (2.1), (3.2), (3.4), (3.5) and (3.6), we obtain (2.2). This completes the proof of part (1).

(2) By the inequality in Osipov and Petrov [9], there exists a bounded and decreasing function $\psi(u)$ on the interval $(0, \infty)$ such that $\lim _{u \rightarrow \infty} \psi(u)=0$ and

$$
\left|P\left(\frac{1}{\sqrt{n} \sigma} S_{n}<x\right)-\Phi(x)\right| \leq \frac{\psi(\sqrt{n}(1+|x|))}{n^{\delta / 2}(1+|x|)^{2+\delta}} .
$$

Let $x=n^{(2-p) / 2 p} \epsilon$, we have $\left|R_{n}\right| \leq \frac{2 \psi\left(\sqrt{n}\left(1+n^{(2-p) / 2 p} \epsilon\right)\right)}{n^{\delta / 2}\left(1+n^{(2-p) / 2 p} \epsilon\right)^{2+\delta}}$, so that:

(a) If $2<r<(1+\delta / 2) p$, then

$$
\epsilon^{2(r-p) /(2-p)} \sum_{n=1}^{\infty} n^{r / p-2} R_{n} \leq \epsilon^{2(r-p) /(2-p)} \sum_{n=1}^{\infty} n^{r / p-2-\delta / 2}=O\left(\epsilon^{2(r-p) /(2-p)}\right) .
$$


(b) If $(1+\delta / 2) p<r<2+\delta$, then by noticing that $\lim _{u \rightarrow \infty} \psi(u)=0$ for any $\eta>0$, there exists a natural number $N_{0}$ such that $\psi(\sqrt{n})<\eta$ whenever $n>N_{0}$. We conclude that

$$
\begin{aligned}
& \epsilon^{2(r-p) /(2-p)} \sum_{n=1}^{\infty} n^{r / p-2} R_{n} \\
& \leq C \epsilon^{2(r-p) /(2-p)} \sum_{n=1}^{\infty} \frac{2 n^{r / p-2} \psi\left(\sqrt{n}\left(1+n^{(2-p) / 2 p} \epsilon\right)\right)}{n^{\delta / 2}\left(1+\epsilon n^{(2-p) / 2 p}\right)^{2+\delta}} \\
& \leq C \epsilon^{2(r-p) /(2-p)}\left(\sum_{n=1}^{N_{0}} n^{r / p-2-\delta / 2} \psi(\sqrt{n})+\eta \sum_{n=N_{0}+1}^{\left[\epsilon^{-2 p /(2-p)}\right]} n^{r / p-2-\delta / 2}\right) \\
& \quad+C \epsilon^{2(r-p) /(2-p)-2-\delta} \psi\left(\epsilon^{-p /(2-p)}\right) \quad \sum_{n=\left[\epsilon^{-2 p /(2-p)]+1}\right.}^{\infty} n^{r / p-2-\delta / 2-(1 / p-1 / 2)(2+\delta)} \\
& \leq \epsilon^{2(r-p) /(2-p)} N_{0}^{r / p-1-\delta / 2}+C \eta \epsilon^{p \delta /(2-p)}+C \psi\left(\epsilon^{-p /(2-p)}\right) \epsilon^{p \delta /(2-p)} \\
&= o\left(\epsilon^{p \delta /(2-p)}\right) .
\end{aligned}
$$

(c) If $r=(1+\delta / 2) p$, then

$$
\epsilon^{2(r-p) /(2-p)} \sum_{n=1}^{\infty} n^{r / p-2} R_{n}=O\left(\epsilon^{p \delta /(2-p)} \log \frac{1}{\epsilon}\right)
$$

By (2.1) and combining with (3.2), (3.7), (3.8) and (3.9), we obtain (2.3), which completes the proof of part (2).

(3) We make use of the following large deviation estimate in Petrov [10]:

$$
\left|P\left(\frac{1}{\sqrt{n} \sigma} S_{n}<x\right)-\Phi(x)\right| \leq \frac{C}{\sqrt{n}(1+|x|)^{q}}, \quad x>0 .
$$

So, $\left|R_{n}\right| \leq \frac{C}{\sqrt{n}\left(1+\epsilon n^{(2-p) / 2 p}\right)^{q}}$. Hence we have the following.

(a) If $r<3 p / 2$, then

$$
\epsilon^{2(r-p) /(2-p)} \sum_{n=1}^{\infty} n^{r / p-2} R_{n} \leq \epsilon^{2(r-p) /(2-p)} \sum_{n=1}^{\infty} n^{r / p-5 / 2}=O\left(\epsilon^{2(r-p) /(2-p)}\right) .
$$

(b) If $r>3 p / 2$, then $\frac{r}{p}-\frac{5}{2}-\frac{2 q-p q}{2 p}<-1$. By noting that $q>\frac{2 r-3 p}{2-p}$, we obtain

$$
\begin{aligned}
\epsilon^{2(r-p) /(2-p)} \sum_{n=1}^{\infty} n^{r / p-2} R_{n} \leq & C \epsilon^{2(r-p) /(2-p)} \sum_{n=1}^{\infty} \frac{n^{r / p-2}}{\left(1+\epsilon n^{(2-p) / 2 p}\right) q \sqrt{n}} \\
\leq & C \epsilon^{2(r-p) /(2-p)} \sum_{n=1}^{\left[\epsilon^{-2 p /(2-p)}\right]} n^{r / p-2-1 / 2} \\
& +C \epsilon^{2(r-p) /(2-p)-q} \sum_{n=\left[\epsilon^{-2 p /(2-p)]+1}\right.}^{\infty} n^{r / p-2-1 / 2-(2-p) q / 2 p} \\
= & O\left(\epsilon^{p /(2-p)}\right) .
\end{aligned}
$$


(c) If $r=3 p / 2$, then

$$
\begin{aligned}
\epsilon^{2(r-p) /(2-p)} \sum_{n=1}^{\infty} n^{r / p-2} R_{n} & \leq C \epsilon^{p /(2-p)} \sum_{n=1}^{\left[\epsilon^{-2 p /(2-p)]}\right.} \frac{1}{n}+C \epsilon^{p /(2-p)-q} \sum_{n=\left[\epsilon^{-2 p /(2-p)]+1}\right.}^{\infty} n^{-1-(2-p) q / 2 p} \\
& =O\left(\epsilon^{p /(2-p)} \log \frac{1}{\epsilon}\right)
\end{aligned}
$$

By (2.1), from (3.2), (3.10), (3.11) and (3.12), we have (2.4), which completes the proof of part (3).

Proof of Theorem 2.2 We write

$$
\begin{aligned}
\epsilon^{2} \lambda_{2,1 /(\beta+1)}(\epsilon)-\frac{1}{2 \beta+1} \\
=\left(\frac{2 \epsilon^{2}}{\sqrt{2 \pi}} \sum_{n=1}^{\infty} n^{2 \beta} \int_{\epsilon n^{\beta+1 / 2}}^{\infty} e^{-t^{2} / 2} d t-\frac{1}{2 \beta+1}\right) \\
\quad+\epsilon^{2}\left(\sum_{n=1}^{\left[\epsilon \epsilon^{-4 /(2 \beta+1)]}\right.}+\sum_{n=\left[\epsilon^{-4 /(2 \beta+1)]+1}\right.}^{\infty}\right) n^{2 \beta}\left(P\left(\left|S_{n}\right| \geq \epsilon n^{\beta+1}\right)-\frac{2}{\sqrt{2 \pi}} \int_{\epsilon n^{\beta+1 / 2}}^{\infty} e^{-t^{2} / 2} d t\right) \\
=: I_{1}+I_{2}+I_{3} .
\end{aligned}
$$

First, according to Lemma 2.1, we have

$$
I_{1}= \begin{cases}O\left(\epsilon^{2}\right), & -\frac{1}{2}<\beta<\frac{1}{2} \\ O\left(\epsilon^{4 /(2 \beta+1)}\right), & \beta \geq \frac{1}{2}\end{cases}
$$

For $I_{3}$, applying Lemma 2.3 of Xie and He [11], and letting $x=2 y=n^{\beta+1} \epsilon$, we obtain

$$
P\left(\left|S_{n}\right| \geq n^{\beta+1} \epsilon\right) \leq n P\left(|X| \geq \frac{1}{2} n^{\beta+1} \epsilon\right)+8 e^{2} \epsilon^{-4} n^{-4 \beta-2} .
$$

Observing the following fact

$$
\frac{2}{\sqrt{2 \pi}} \int_{\epsilon n^{\beta+1 / 2}}^{\infty} e^{-t^{2} / 2} d t=2\left(1-\Phi\left(n^{\beta+\frac{1}{2}} \epsilon\right)\right) \leq \frac{2 \varphi\left(n^{\beta+1 / 2} \epsilon\right)}{n^{\beta+1 / 2} \epsilon}=O\left(\epsilon^{-5} n^{-5 \beta-5 / 2}\right),
$$

from (3.15) and (3.16), we have

$$
\begin{aligned}
I_{3} \leq & \epsilon^{2} \sum_{n=\left[\epsilon^{-4 /(2 \beta+1)}\right]+1}^{\infty} n^{2 \beta} P\left(\left|S_{n}\right| \geq \epsilon n^{\beta+1}\right)+\epsilon^{2} \sum_{n=\left[\epsilon^{-4 /(2 \beta+1)}\right]+1}^{\infty} \frac{2 n^{2 \beta}}{\sqrt{2 \pi}} \int_{\epsilon n^{\beta+1 / 2}}^{\infty} e^{-t^{2} / 2} d t \\
\leq & \epsilon^{2} \sum_{n=\left[\epsilon^{-4 /(2 \beta+1)}\right]+1}^{\infty} n^{2 \beta+1} P\left(|X|>\frac{\epsilon n^{\beta+1}}{2}\right)+C \epsilon^{-2} \sum_{n=\left[\epsilon^{-4 /(2 \beta+1)}\right]+1}^{\infty} n^{-2 \beta-2} \\
& +C \epsilon^{-3} \sum_{n=\left[\epsilon^{-4 /(2 \beta+1)}\right]+1}^{\infty} n^{-3 \beta-5 / 2}
\end{aligned}
$$




$$
\begin{aligned}
& \leq \epsilon^{2} \sum_{n=\left[\epsilon^{-4 /(2 \beta+1)}\right]+1}^{\infty} n^{2 \beta+1} \int_{|x| \geq \frac{1}{2} n^{\beta+1} \epsilon} d F(x)+O\left(\epsilon^{2}\right)+O\left(\epsilon^{3}\right) \\
& \leq \epsilon^{2} \sum_{n=\left[\epsilon^{-4 /(2 \beta+1)}\right]+1}^{\infty} n^{2 \beta+1} \sum_{k=n}^{\infty} \int_{\frac{1}{2} k^{\beta+1} \epsilon \leq x<\frac{1}{2}(k+1)^{\beta+1} \epsilon} d F(x)+O\left(\epsilon^{2}\right) \\
& \leq \epsilon^{2} \sum_{k=\left[\epsilon^{-4 /(2 \beta+1)}\right]+1}^{\infty} \sum_{n=1}^{k} n^{2 \beta+1} \int_{\frac{1}{2} k^{\beta+1} \epsilon \leq x<\frac{1}{2}(k+1)^{\beta+1} \epsilon} d F(x)+O\left(\epsilon^{2}\right) \\
& \leq C \epsilon^{2} \sum_{k=\left[\epsilon^{-4 /(2 \beta+1)}\right]+1}^{\infty} k^{2 \beta+2} \int_{\frac{1}{2} k^{\beta+1} \epsilon \leq x<\frac{1}{2}(k+1)^{\beta+1} \epsilon} d F(x)+O\left(\epsilon^{2}\right) \\
& \leq C \sum_{k=\left[\epsilon^{-4 /(2 \beta+1)}\right]+1}^{\infty} \int_{\frac{1}{2} k^{\beta+1} \epsilon \leq x<\frac{1}{2}(k+1)^{\beta+1} \epsilon} x^{2} d F(x)+O\left(\epsilon^{2}\right) \\
& \leq C \int_{x \geq \frac{1}{2} \epsilon^{-(2 \beta+3) /(2 \beta+1)}} x^{2} d F(x)+O\left(\epsilon^{2}\right) \\
& =C T\left(\epsilon^{-(2 \beta+3) /(2 \beta+1)}\right)+O\left(\epsilon^{2}\right) .
\end{aligned}
$$

Using the assumption on $T(t)$ and Lemma 2.2(1), we can obtain

$$
I_{3}= \begin{cases}O\left(\epsilon^{2}\right), & -\frac{1}{2}<\beta \leq \frac{\min (\delta, 1)}{4}-\frac{1}{2}, \\ O\left(\epsilon^{1 /(2 \beta+1)}\right), & \beta \geq-\frac{1}{4}, \delta \geq 1, \\ O\left(\epsilon^{\delta /(2 \beta+1)} l\left(\epsilon^{-1 /(2 \beta+1)}\right)\right), & \beta \geq-\frac{1}{2}+\frac{\delta}{4}, 0<\delta<1 .\end{cases}
$$

For $I_{2}$, by Bikelis's inequality (see [12]), we have

$$
\begin{aligned}
I_{2} \leq & \epsilon^{2} \sum_{n=1}^{\left[\epsilon^{-4 /(2 \beta+1)}\right]} \frac{C n^{2 \beta}}{\left(1+\epsilon n^{\beta+1 / 2}\right)^{3} \sqrt{n}} \int_{0}^{\left(1+\epsilon n^{\beta+1 / 2}\right) \sqrt{n}} T(v) d v \\
\leq & \epsilon^{2} \sum_{n=1}^{\left[\epsilon^{-2 /(2 \beta+1)}\right]} n^{2 \beta-1 / 2} \int_{0}^{\left(1+\epsilon n^{\beta+1 / 2}\right) \sqrt{n}} T(v) d v \\
& +\epsilon^{-1} \sum_{n=\left[\epsilon^{-2 /(2 \beta+1)}\right]+1}^{\left[\epsilon^{-4 /(2 \beta+1)]}\right.} n^{-\beta-2} \int_{0}^{\left(1+\epsilon n^{\beta+1 / 2}\right) \sqrt{n}} T(v) d v .
\end{aligned}
$$

Now, the proof of Theorem 2.2 will be divided into the following cases.

Case 1 of $\delta>1$.

Noting that $T(t) \leq E X^{2}=1$, let $\delta_{1}$ be a real number such that $1<\delta_{1}<\delta$, by Lemma 2.2(1), $\lim _{t \rightarrow \infty} t^{\delta_{1}-\delta} l(t)=0$. Therefore, there is a real number $T_{0}>0$ such that $\left|\frac{l(t)}{t^{\delta-\delta_{1}}}\right|<1$ whenever $t>T_{0}$. Then

$$
\int_{0}^{\infty} T(t) d t \leq \int_{0}^{1} T(t) d t+\int_{1}^{\infty} T(t) d t \leq C+\int_{T_{0}}^{\infty} \frac{1}{t^{\delta_{1}}} d t<\infty
$$


We have

$$
\begin{aligned}
I_{2} & \leq C \epsilon^{2} \sum_{n=1}^{\left[\epsilon^{-2 /(2 \beta+1)}\right]} n^{2 \beta-1 / 2}+C \epsilon^{-1} \sum_{n=\left[\epsilon^{-2 /(2 \beta+1)}\right]+1}^{\left[\epsilon^{-4 /(2 \beta+1)}\right]+1} n^{-\beta-2} \\
& = \begin{cases}O\left(\epsilon^{2}\right), & -\frac{1}{2}<\beta \leq-\frac{1}{4}, \\
O\left(\epsilon^{2} \log \frac{1}{\epsilon}\right), & \beta=-\frac{1}{4}, \\
O\left(\epsilon^{1 /(2 \beta+1)}\right), & \beta>-\frac{1}{4} .\end{cases}
\end{aligned}
$$

From (3.13), (3.14), (3.17) and (3.18), we obtain (2.5).

Case 2 of $0<\delta<1$.

(a) If $-\frac{1}{2}<\beta<-\frac{1}{2}+\frac{\delta}{4}$, then $\sum_{n=1}^{\infty} n^{2 \beta-1 / 2}<\infty$ and $\int_{1}^{\infty} t^{4 \beta+1-\delta} l(t) d t<\infty$. Making use of Lemma 2.2(2)-(3), we have

$$
\begin{aligned}
& I_{2} \leq C \epsilon^{2} \sum_{n=1}^{\left[\epsilon^{-2 /(2 \beta+1)}\right]} n^{2 \beta-1 / 2}\left(1+\int_{1}^{2 \sqrt{n}} T(t) d t\right) \\
& +C \epsilon^{-1} \sum_{n=\left[\epsilon^{-2 /(2 \beta+1)}\right]+1}^{\left[\epsilon^{-4 /(2 \beta+1)}\right]} n^{-\beta-2}\left(1+\int_{1}^{2 \epsilon n^{\beta+1}} T(t) d t\right) \\
& \leq C \epsilon^{2} \sum_{n=1}^{\left[\epsilon^{-2 /(2 \beta+1)}\right]} n^{2 \beta-1 / 2}(\sqrt{n})^{1-\delta} l(\sqrt{n})+O\left(\epsilon^{2}\right) \\
& +C \epsilon^{1 /(2 \beta+1)}+C \epsilon^{-1} \sum_{n=\left[\epsilon^{-2 /(2 \beta+1)}\right]+1}^{\left[\epsilon^{-4 /(2 \beta+1)}\right]} n^{-\beta-2}\left(2 n^{\beta+1} \epsilon\right)^{1-\delta} l\left(2 n^{\beta+1} \epsilon\right) \\
& \leq C \epsilon^{2} \int_{1}^{\epsilon^{-2 /(2 \beta+1)}} x^{2 \beta-1 / 2}(\sqrt{x})^{1-\delta} l(\sqrt{x}) d x \\
& +C \epsilon^{-\delta} \int_{\epsilon^{-2 /(2 \beta+1)}}^{\infty} x^{-\beta-2} l\left(2 x^{\beta+1} \epsilon\right) x^{(\beta+1)(1-\delta)} d x+O\left(\epsilon^{2}\right) \\
& \leq C \epsilon^{2} \int_{1}^{\epsilon^{-1 /(2 \beta+1)}} t^{4 \beta+1-\delta} l(t) d t+C \int_{\epsilon^{-1 /(2 \beta+1)}}^{\infty} \frac{l(t)}{t^{1+\delta}} d t+O\left(\epsilon^{2}\right) \\
& \leq C \epsilon^{2}+C \epsilon^{\delta /(2 \beta+1)} l\left(\epsilon^{-1 /(2 \beta+1)}\right) \\
& =O\left(\epsilon^{2}\right) \text {. }
\end{aligned}
$$

(b) If $\beta \geq-\frac{1}{2}+\frac{\delta}{4}$, then we have

$$
\begin{aligned}
I_{2} & \leq C \epsilon^{2} \epsilon^{-(4 \beta+1) /(2 \beta+1)}\left(1+\int_{1}^{2 \epsilon^{-1 /(2 \beta+1)}} T(t) d t\right)+C \epsilon^{1 /(2 \beta+1)}+C \epsilon^{\delta /(2 \beta+1)} l\left(\epsilon^{-1 /(2 \beta+1)}\right) \\
& \leq C \epsilon^{1 /(2 \beta+1)}\left(1+\left(2 \epsilon^{-(1-\delta) /(2 \beta+1)} l\left(\epsilon^{-1 /(2 \beta+1)}\right)\right)+C \epsilon^{\delta /(2 \beta+1)} l\left(\epsilon^{-1 /(2 \beta+1)}\right)\right. \\
& \leq C \epsilon^{\delta /(2 \beta+1)} l\left(\epsilon^{-1 /(2 \beta+1)}\right) .
\end{aligned}
$$


Therefore

$$
I_{2}= \begin{cases}O\left(\epsilon^{2}\right), & -\frac{1}{2}<\beta \leq-\frac{1}{2}+\frac{\delta}{4}, \\ O\left(\epsilon^{\delta /(2 \beta+1)} l\left(\epsilon^{1 /(2 \beta+1)}\right)\right), & \beta \geq-\frac{1}{2}+\frac{\delta}{4} .\end{cases}
$$

Combining the estimate with (3.11) and (3.14), by (3.10), this implies that (2.6) follows.

Case 3 of $\delta=1$.

(a) If $-\frac{1}{2}<\beta<-\frac{1}{4}$, then $\sum_{n=1}^{\infty} n^{2 \beta-\frac{1}{2}}<\infty$. We have

$$
\begin{aligned}
I_{2} \leq & C \epsilon^{2}\left(1+\int_{1}^{\epsilon^{-1 /(2 \beta+1)}} T(t) d t\right) \sum_{n=1}^{\left[\epsilon^{-2 /(2 \beta+1)}\right]} n^{2 \beta-1 / 2} \\
& +C \epsilon^{-1}\left(1+\int_{1}^{\epsilon^{-\frac{2 \beta+3}{2 \beta+1}}} T(t) d t\right) \sum_{n=\left[\epsilon^{-2 /(2 \beta+1)}\right]+1}^{\left[\epsilon^{-4 /(2 \beta+1)}\right]} n^{-\beta-2} \\
\leq & C \epsilon^{2}\left(1+\int_{1}^{-1 /(2 \beta+1)} \frac{l(t)}{t} d t\right)+\epsilon^{1 /(2 \beta+1)}\left(1+\int_{1}^{\epsilon^{-\frac{2 \beta+3}{2 \beta+1}}} \frac{l(t)}{t} d t\right) \\
\leq & C \epsilon^{2}\left(1+\int_{1}^{\epsilon^{-1 /(2 \beta+1)}} \frac{l(t)}{t} d t\right) .
\end{aligned}
$$

(b) If $\beta>-\frac{1}{4}$, then we have

$$
\begin{aligned}
I_{2} & \leq C \epsilon^{2} \epsilon^{\frac{-2}{2 \beta+1}(2 \beta+1 / 2)}\left(1+\int_{1}^{\epsilon^{-1 /(2 \beta+1)}} \frac{l(t)}{t} d t\right)+C \epsilon^{1 /(2 \beta+1)}\left(1+\int_{1}^{\epsilon^{-(2 \beta+3) /(2 \beta+1)}} \frac{l(t)}{t} d t\right) \\
& \leq C \epsilon^{1 /(2 \beta+1)}\left(1+\int_{1}^{-1 /(2 \beta+1)} \frac{l(t)}{t} d t\right)+\epsilon^{1 /(2 \beta+1)}\left(1+\int_{1}^{\epsilon^{-(2 \beta+3) /(2 \beta+1)}} \frac{l(t)}{t} d t\right) \\
& \leq C \epsilon^{1 /(2 \beta+1)}\left(1+\int_{1}^{\epsilon^{-(2 \beta+3) /(2 \beta+1)}} \frac{l(t)}{t} d t\right) .
\end{aligned}
$$

(c) If $\beta=-\frac{1}{4}$, then we have

$$
\begin{aligned}
I_{2} & \leq C \epsilon^{2} \log \frac{1}{\epsilon}\left(1+\int_{1}^{\epsilon^{-2}} \frac{l(t)}{t} d t\right)+C \epsilon^{2}\left(1+\int_{1}^{\epsilon^{-5}} \frac{l(t)}{t} d t\right) \\
& \leq C \epsilon^{2} \log \frac{1}{\epsilon}\left(1+\int_{1}^{\epsilon^{-5}} \frac{l(t)}{t} d t\right)
\end{aligned}
$$

so that

$$
I_{2}= \begin{cases}O\left(\epsilon^{2}\left(1+\int_{1}^{\epsilon^{-1 /(2 \beta+1)}} \frac{l(t)}{t} d t\right)\right), & -\frac{1}{2}<\beta \leq-\frac{1}{4}, \\ O\left(\epsilon^{2}\left(1+\int_{1}^{\epsilon^{-5}} \frac{l(t)}{t} d t\right) \log \frac{1}{\epsilon}\right), & \beta=-\frac{1}{4}, \\ O\left(\epsilon^{1 /(2 \beta+1)}\left(1+\int_{1}^{\epsilon^{-(2 \beta+3) /(2 \beta+1)}} \frac{l(t)}{t} d t\right)\right), & \beta>-\frac{1}{4} .\end{cases}
$$

Combining the estimate with (3.14) and (3.17), by (3.13), this implies that (2.7) follows, and hence Theorem 2.2 is proved. 
Proof of Theorem 2.3 Set $T_{1}(t)=E|X|^{2+\delta} I(|X|>t)$. First, note that

$$
\begin{aligned}
E|X|^{2+\delta} I(|X|>t) & =\int_{|x|>t}|x|^{2+\delta} d F(x) \\
& =\int_{|x|>t} x^{2}\left(\int_{t}^{|x|} \delta y^{\delta-1} d y\right) d F(x)+t^{\delta} \int_{|x|>t} x^{2} d F(x) \\
& =\int_{t}^{\infty} \delta y^{\delta-1}\left(\int_{|x|>y} x^{2} d F(x)\right) d y+t^{\delta} T(t) \\
& =\delta \int_{t}^{\infty} s^{\delta-1} T(s) d s+t^{\delta} T(t) .
\end{aligned}
$$

We have

$$
T_{1}(t)=\delta \int_{t}^{\infty} s^{\delta-1} T(s) d s+t^{\delta} T(t) .
$$

Since $\int_{t}^{\infty} s^{\delta-1} T(s) d s \geq 0, t^{\delta} T(t) \geq 0$, we have

$$
T_{1}(t) \rightarrow 0 \quad \Leftrightarrow \quad t^{\delta} T(t) \rightarrow 0 \quad \text { and } \quad \int_{t}^{\infty} s^{\delta-1} T(s) d s \rightarrow 0 \quad \text { as } t \rightarrow \infty \text {. }
$$

Next, it is easy to get

$$
E|X|^{2+\delta}<\infty \quad \Leftrightarrow \quad T_{1}(t) \rightarrow 0 \quad \text { as } t \rightarrow \infty .
$$

From the above facts, the proof of Theorem 2.3 is complete.

\section{Competing interests}

The author declares that they have no competing interests.

\section{Acknowledgements}

The author would like to thank the referee for helpful comments.

Received: 3 February 2013 Accepted: 18 July 2013 Published: 11 August 2013

\section{References}

1. Heyde, CC: A supplement to the strong law of large numbers. J. Appl. Probab. 12, 903-907 (1975)

2. Klesov, Ol: On the convergence rate in a theorem of Heyde. Theory Probab. Math. Stat. 49, 83-87 (1994)

3. He, JJ, Xie, TF: Asymptotic property for some series of probability. Acta Math. Appl. Sin. 29, 179-186 (2013)

4. Gut, A, Steinebach, J: Convergence rates in precise asymptotics. J. Math. Anal. Appl. 390, 1-14 (2012)

5. Gut, A, Steinebach, J: Precise asymptotics-a general approach. Acta Math. Hung. 138, 365-385 (2013)

6. Gut, A, Steinebach, J: Correction to 'Convergence rates in precise asymptotics'. Preprint (http://www2.math.uu.se/ allan/90correction.pdf) (2012)

7. Bingham, NH, Goldie, CM, Teugels, JL: Regular Variation. Cambridge University Press, Cambridge (1987)

8. Nagaev, SV: Some limit theorems for large deviation. Theory Probab. Appl. 10, 214-235 (1965)

9. Osipov, LV, Petrov, WV, On an estimate of remainder term in the central limit theorem. Theory Probab. Appl. 12, 281-286 (1967)

10. Petrov, W: Limit Theorems of Probability Theory. Oxford University Press, Oxford (1995)

11. Xie, TF, He, JJ: Rate of convergence in a theorem of Heyde. Stat. Probab. Lett. 82, 1576-1582 (2012)

12. Bikelis, A: Estimates of the remainder in the central limit theorem. Liet. Mat. Rink. 6, 323-346 (1966)

doi:10.1186/1029-242X-2013-378

Cite this article as: He: A note to the convergence rates in precise asymptotics. Journal of Inequalities and Applications $20132013: 378$ 